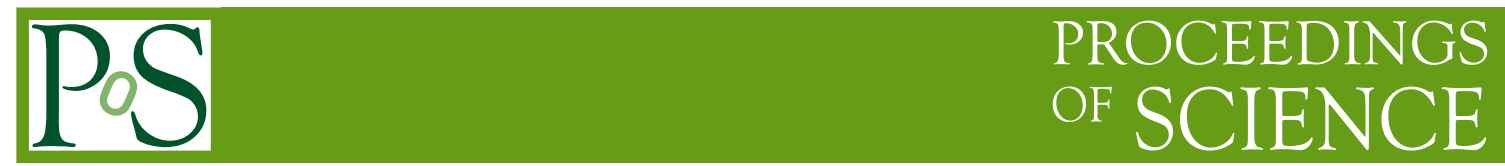

\title{
How do Pulsars blow their Winds?
}

\section{Wolfgang Kundt*}

Argelander Institut of Bonn University, Germany

E-mail: wkundteastro.uni-bonn.de

Pulsars are known to blow strong winds, via their occasional bow shocks, via all their (coherent and incoherent) radiation, and more indirectly via their acceleration of (most of) the Cosmic Rays, and via their emission of (all) the $\gamma$-ray bursts (GRBs). But the precise mechanism of how they do this is still controversial, to say the least. A new approach to this difficult problem will be proposed.

XI Multifrequency Behaviour of High Energy Cosmic Sources Workshop 25-30 May 2015

Palermo, Italy

${ }^{*}$ Speaker. 


\section{Background Knowledge about Winds from Neutron Stars}

Our knowledge about pulsar winds is only indirect: via their occasional bowshocks, via the pulsed radiation of hundreds of Galactic neutron stars - from radio signals up in frequency to hard $\gamma$-ray energies - via supernova remnants (SNRs), and probably also via all the $\gamma$-ray bursts (GRBs, [Kundt, 2014]), and as the dominant boosters of the cosmic rays (CRs, [Kundt, 2012]). But in my understanding, the literature of the past 45 years since their discovery does not contain a viable explanation of how the pulsars blow their strong, relativistic winds.

Neutron-star surface matter is gravitationally bound at $\approx 10^{-1}$ of its rest energy, so that thermal forces are too weak to extract a wind. But electrical forces, e $\beta_{c o r} \times \mathbf{B},\left(\beta_{c o r}:=\mathrm{R} \Omega / \mathrm{c}\right)$, exerted by their corotating magnetosphere, are some $10^{13}$ times stronger than gravitational ones, $\mathrm{mg}$, hence can easily extract charges of the correct sign, whereby the corresponding charges of the opposite sign are boosted downwards towards the surface, so that the star charges up oppositely in sign to the ejected charge layer. Such electric extractions can thus last only for roughly half a sparking period $\mathrm{P}_{s p}$, of duration $\sqrt{\mathrm{R}^{3} \Omega / \mathrm{c}^{3}} \gtrsim$ microsecond, i.e. for a tiny fraction of the star's spin period, and must occur periodically if a quasi-steady wind is to flow. And they must be strongly enhanced in order to reach the wind strengths inferred from the observations. Ever since the pioneering work of Ruderman \& Sutherland, such enhancements have been thought to be achieved in flight by the extracted primary charges via collisional $\mathrm{e}^{ \pm}$-pair formations, both during collisions with hard photons, via curvature radiation, and during collisions with transverse $\mathbf{B}$-fields, by the Erber mechanism. Doubts remained, however, how the outgoing electric currents could be closed, and whether or not the achievable neutral pair excesses were strong enough to yield the observed wind strengths.

The best estimate of the (enormous) pulsar wind strengths is obtained from their (occasional) bowshocks - in transit through denser regions of the Galactic ISM - whose momentum balance wants large neutral wind excesses $\xi\left(:=\mathrm{n} / \mathrm{n}_{\text {cor }}\right.$ ) over the (minimum) Goldreich-Julian number densities $\mathrm{n}_{\text {cor }}\left(=\Omega \mathbf{B} \gamma_{\text {cor }}^{2} / 2 \pi \mathrm{ce}\right)$, of order $\xi \approx 10^{4}$. Here comes my new wind injection mechanism, whose importance I have overlooked in the past, ever since [Kundt \& Schaaf, 1993], and [Kundt, 1998, 2004]: Whilst some quasi-spherical charge layer is ejected by the outward-pointing GoldreichJulian E-field, its counter layer (of opposite sign) is shot downward onto the stellar surface where it collisionally creates a dense excess layer of extremely relativistic $\mathrm{e}^{ \pm}$-pairs, a large fraction of which escapes in all directions, immediately after formation, in essential ignorance of the strong magnetic field. They form a dense, almost neutral, relativistic wind layer whose bulk motion is only controlled by gravity. This wind escapes, therefore, from the whole surface of the neutron star - not only from its polar caps - without any post-acceleration, without inner and outer gaps, and without corotating regions, but likewise with strong post-acceleration beyond the speed-of-light cylinder (SLC) where $\mathbf{E} \times \mathbf{B}$-forces push both electrons and positrons radially outward, in the same direction. What was called "pair corona" in [1993] is now replaced by regularly varying layers of (mildly charged) pair plasma near the surface, partially from the escaping wind, but predominantly from its trailing 'wake', with less than escape-speed. I have called these repetitive thin layers of escaping pair-plasma in the windzone "chapátis", in acknowledgement of Ashok Singal's multiple contributions to earlier studies in this field.

Once more, in slightly different words: Pulsar winds are (claimed to be) launched by thin, 
downward boosted electron layers of alternating sign, bombarding the stellar surface periodically on the sparking timescale $\mathrm{P}_{s p} / 2$. In this way, (alternatingly charged) chapátis escape successively at almost the speed of light, forming a pulsar's strong, on average neutral, relativistic wind. For comparison with the recent work of other people, the reader is referred to the (predominantly numerical) approach by Chen \& Beloborodov [2014].

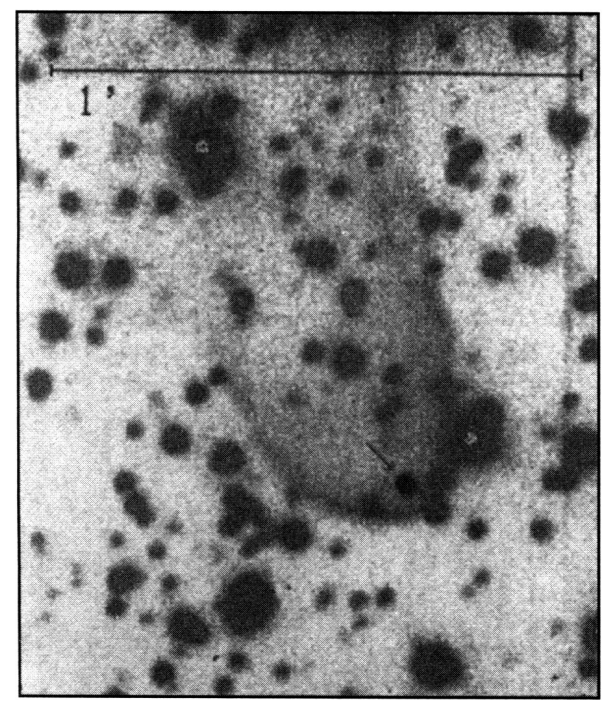

Figure 1: $\mathrm{H} \alpha$ map of the msec-PSR $1957+20$ moving at $10^{2}(\mathrm{~km} / \mathrm{s})(\mathrm{d} / 0.73 \mathrm{kpc})$ through the ISM, mapped by Kulkarni \& Hester [1988]. Bar length $=1^{\prime} \simeq 0.22 \mathrm{pc}(\mathrm{d} / 0.73 \mathrm{kpc})$.

Before returning to the new wind-generation mechanism, allow me to stress the relevance of two long-standing observations on pulsars: Fig. 1 is an $\mathrm{H} \alpha$ map proving the strength of the bowshock of the msec-PSR B1957+20 as it ploughs through the nearby ISM: Pulsar winds can exert significant ram pressures, despite being relativistic. We only observe such clear bowshocks in suitable regions of the (inhomogeneous) ISM, cool enough for HI emission.

Fig. 2 plots the logarithmic mean-field intensity profiles of two pulsars sampled at high dynamic range. It shows that on a logarithmic scale, pulsars radiate essentially at all angles, not only at fixed spin phases. Their radio pulses are thought to be emitted coherently, with typically some $\mathrm{N} \approx 10^{14}$ electrons radiating in phase, so that their intensities scale as $\mathrm{N}^{2}\left(\approx 10^{28}\right)$. Clearly, small fluctuations in $\mathrm{N}(\varphi, \mathrm{t})$ give rise to largely fluctuating intensities $\mathrm{I}(\mathrm{t})$, i.e. to time-varying intensities ranging through many powers of ten.

\section{The New Proposal}

As already stressed, pulsar winds require powerful formation which we identify as oscillatory surface bombardment, (not steady ejection of charges). Such escape must not be of dipolar geometry, rather more isotropic, and even locally on average free of electric currents, without global return currents. Surface bombardment guarantees ample supply of wind substance: $\xi \approx 10^{4}$. Whereby the controlling forces are electric, of the form $\mathbf{E} \sim \beta \times \mathbf{B}$; they scale as $\mathrm{B} / \mathrm{P}$ with varying magnetic field strength $\mathrm{B}$ and spin period $\mathrm{P}$, hence are fairly uniform for the complete set of pulsars. The 

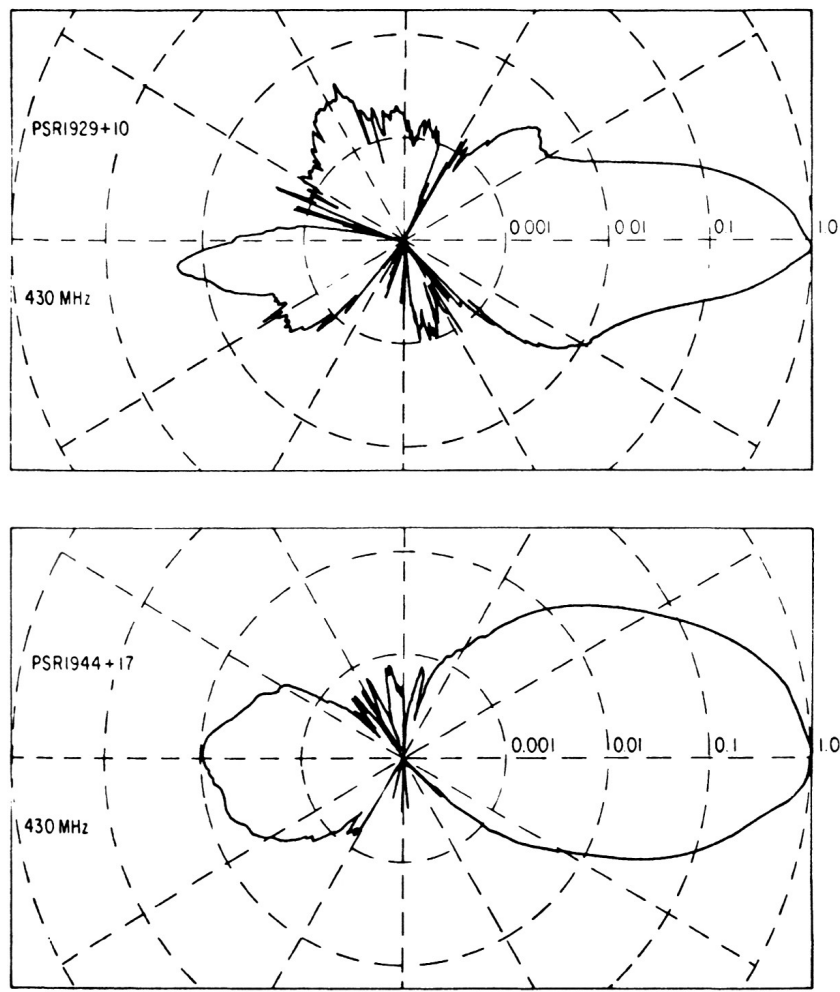

Figure 2: Logarithmic mean-pulse intensity profiles of two pulsars, showing how wide in phase such profiles can extend when sampled at sufficient $\left(\lesssim 10^{-3}\right)$ dynamic range; [Hankins \& Fowler, 1986].

whole stellar surface emits the wind, in order to guarantee wide-angle radio emission (on a logarithmic scale, fig. 2). And the winds are all post-accelerated, by the outgoing low-frequency waves from their corotating magnetosphere, with their strong, charge-independent $\mathbf{E} \times \mathbf{B}$-force.

These aspects are indicated in the cartoon of Fig. 3, which may help identifying them. Crucial for successful blowing is a minimal thrust of the wind, to overcome the ambient plasma pressure, and to blow a cavity [Kundt, 2004, pp.104-108]; it controls the active lifetime of a pulsar, of order $10^{6.4} \mathrm{yr}$ for typical Galactic environments, but longer inside (hot) globular clusters, yet vanishingly short in the Galaxy's high-pressure core, of (angular) extent $1^{0}$ around Sgr A*, (inside of which no pulsar has been detected yet, though many hundreds of them are expected). Pulsars stop their permanent radio-pulse emissions once their cavity has collapsed, but they continue radiating at $\mathrm{X}$-ray and higher frequencies, via accreting plasma flows.

So how does a pulsar launch its wind? As a rotating magnet, a pulsar necessarily switches on its 'coronal' electric field $\mathbf{E} \sim \beta \times \mathbf{B}$, everywhere above its surface, of uniform direction in large subdomains of its corotating magnetosphere, whereupon stray charges are boosted to extremely relativistic speeds, both 'upward' and 'downward' w.r.t. its surface, with the downward ones bombarding the surface, thereby creating layers of relativistic pair plasma. The bombardment also charges the surface, transiently, at least patchwise. A permanent, on average neutral (pair plasma) wind can only flow if in addition, a residual, quasi-uniform charge layer forms near the surface such that the original, oscillatory coronal $\mathbf{E}$-field is compensated by it when averaged over one 


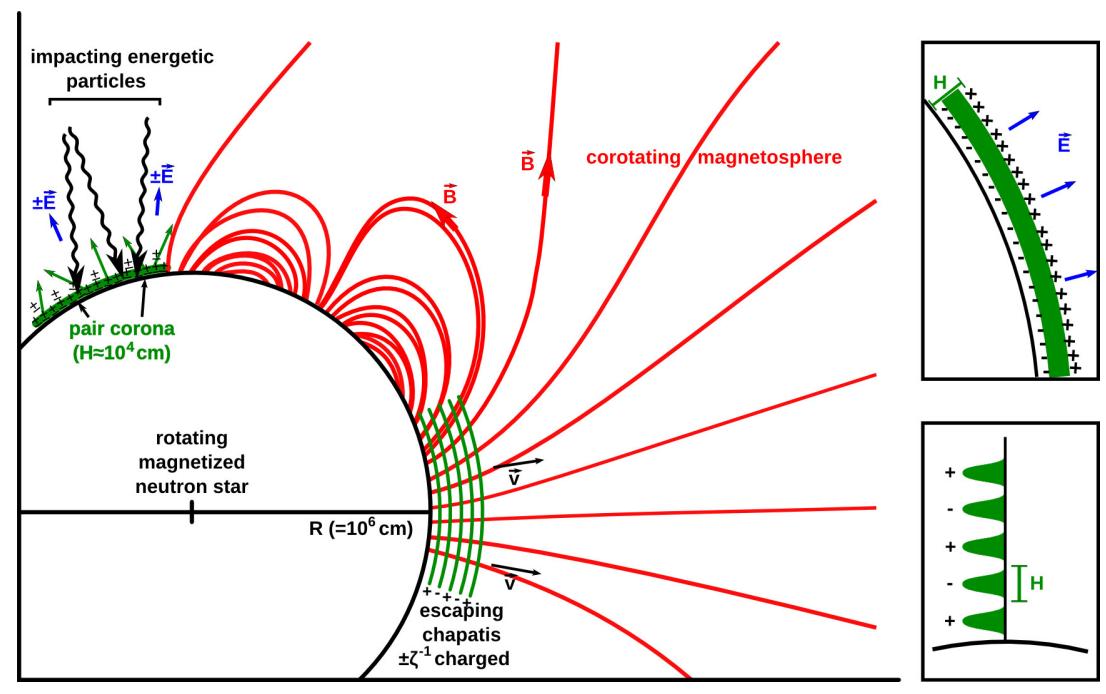

Figure 3: Cartoon of a pulsar in action, indicating variants of the expected multipolar magnetosphere structure (in red), charged surface bombardment and escape (in black), and escaping alternatingly charged pairplasma layers (chapátis) (in green). A realistic description of its wind production should not omit any of these processes.

cycle. In this way, the total accelerating $\mathbf{E}$-field - the sum of the oscillatory coronal field plus its compensating, quasi constant $\mathbf{E}$-field - make sure that the surface is alternatingly bombarded by charged 'downward' electron layers with on average net charge zero, and that oppositely charged electron layers escape towards infinity and form the - on average neutral - wind of the pulsar. The (alternatingly) weakly charged electron layers are called chapátis in Fig. 3; their ram pressure must exceed the ambient CSM pressure, still after having climbed out of the neutron star's strong gravity field, in order for it to blow a windzone of radius $\gtrsim 10^{15} \mathrm{~cm}$, i.e. in order to function as a pulsar.

And how does this new interpretation explain a pulsar's radiation, from radio frequencies up all the way to hard $\gamma$-rays? According to fundamental physics, the mean lever arm of pulsar radiation must equal its speed-of-light distance $\mathrm{c} / \Omega$ [Kundt, 2004, p.105]. Mainly for this reason - but likewise consistent with everything else we know about intensities and phases of broadband pulsar radiation - I expect most of their radio emission to reach us from inside the speed-of-light distance, at high coherence, but all harder radiation incoherently from outside the speed-of-light distance. This general understanding does not change for the new wind model, nor does the inverseCompton explanation change for all the incoherent emissions from the distant wind zone. New for the present wind-formation model is the mode of emission of their coherent radio waves.

How can the new wind-formation model explain the emission of the pulsar's strong, coherent radio waves? This non-trivial problem had not even been solved uncontroversially in earlier approaches. In [Kundt,1998], it was approached with a MAFER ansatz, as 'Microwave Amplification by Forced Emission of Radiation', with details left unanswered for future improvements. I now think that this highly coherent radiation should be understood (newly) as the result of the relativistic pairplasma wind's crossing the pulsar's strong, corotating magnetosphere: During this crossing, electrons and positrons sense opposite $\beta \times \mathbf{B}$-forces, hence are forced to radiate in highly 
polarised ways, both linearly, and circularly, at narrow angles w.r.t. their forward directions. Such forced radiation in almost tangential directions will be strongest at separations (from the pulsar's surface) which are not too large, in order for the wind not to have been too diluted and/or slowed down, but not too small either in order for a sufficient relative speed to have built up w.r.t. the (transversely) rotating magnetosphere. This new mode of highly coherent radiation should be discussed in another publication.

\section{Summary of the Model}

Almost 50 years of international research on pulsars have not sufficed - so we claim - to provide reliable basic equations, or algorithms for a quantitative description of the wind formation by neutron stars, or for secure predictions of their output, both ejections and emissions. In my understanding, neutron stars are not only the most versatile and broadband emitters in the local Universe, but also the dominant generators of the Cosmic Rays, and of the (daily) Gamma-Ray Bursts.

Acknowledgements: My sincere thanks go to Ole Marggraf, Udo Wernick, and Hans Baumann.

\section{References}

[1] Chen, Alexander Y., Beloborodov, Andrei M.: Electrodynamics of Axisymmetric Pulsar Magnetosphere with Electron-Positron discharge: a numerical experiment, The Astrophysical Journal Letters 795: L22 (5pp) 2014.

[2] Hankins, Tim, Fowler, Lee: Astrophys. J. 304, 256 (1986).

[3] Kulkarni, Shri, Hester, J.J.: Nature 335, 801 (1988).

[4] Kundt, W.: Astrophysics of Neutron Stars, Facts and Fiction about their Formation and Functioning, Fundamentals of Cosmic Physics Vol. 20, pp.1-119, 1998.

[5] Kundt, W.: Astrophysics, A New Approach, Springer, 223 pp. (2004).

[6] Kundt, W.: ISM, Cosmic Rays, and the Shape of the Heliosphere, in: MEMORIE S.A.It. 83, pp. 38-44 (2012).

[7] Kundt, W.: Astrophysics without Black Holes, and without Extragalactic Gamma-Ray Bursts, Acta Polytechnica 1(1), 27-33 (2014).

[8] Kundt, W., Marggraf, O.: Physikalische Mythen auf dem Prüfstand, Springer, 445 Seiten (2014).

[9] Kundt, W., Schaaf, R.: Towards Solution of the Pulsar Problem, Astrophysics \& Space Science 200, 251-270 (1993). 


\section{DISCUSSION}

Topinka, Martin: If your GRBs are emitted by our Galaxy, how do you explain the high isotropy of their arrival directions?

Kundt, Wolfgang: Thank you for this relevant question. As stressed e.g. in [7], this isotropy is not perfect at all. But approximate isotropy results - in my understanding - from a combination of a near-spherical (loud) source distribution with preferred radiation in their disk planes, which latter tend to counter-align with the Galactic disk.

Topinka, Martin: In the absence of BHs: How do you explain the existence of two different types of QPO, (which have been explained by two different types of boundary condition: BH horizon vs. solid surface of neutron star)?

Kundt, Wolfgang: Our understanding of QPOs has always been controversial. To me, QPOs look like noisy Kepler speeds. Are they created by oscillations of the corotating magnetosphere, whereby the $\mathrm{n}$ *'s magnetosphere is plasma loaden, and rubs against the inner edge of its accretion disk? BHCs have self-gravitating - and hence almost rigidly rotating - accretion disks, though not near their inner edges, (of small mass density).

Topinka, Martin: If the redshifts in your GRB model are kinematic - indicating transrelativistic velocities of the ejecta - why are the lines not broader?

Kundt, Wolfgang: Again a very good question. The afterglows of my GRBs should be understood as light echos, emitted by CSM along a growing set of confocal ellipsoids, see [8, Fig. 5.4.3]. Whilst the (almost instantaneous) prompt emission is blueshifted, almost all the succeeding (afterglow) radiation comes from the opposite pole of the ellipsoid, with purely radial redshift. Only late afterglows are expected to contain (weak) broad lines. 